\title{
Journal of Clinical and Nursing Research
}

\author{
Research Article
}

\section{Effect of KAP Intervention Model on Resilience and Cancer-related Fatigue in Patients with Colorectal Cancer Undergoing Chemotherapy}

Hui Zhang

Handan Central Hospital, Handan 056000, Hebei Province, China

\begin{abstract}
Objective: To explore the effect of KAP intervention mode on resilience and cancer-related fatigue in patients with colorectal cancer undergoing chemotherapy. Methods: A prospective randomized trial was conducted. 55 patients with colorectal cancer who received routine nursing from February 2018 to February 2019 were included in the control group, and 55 patients who received routine nursing + KAP intervention from March 2019 to March 2020 were included in the observation group. The scores of Resilience Scale and cancer-related fatigue scale (CFS) before and 6 months after intervention were compared between the two groups. Results: After 6 months of intervention, the score of resilience of the two groups was higher than that before intervention, and that of the observation group was higher than that of the control group, the difference was statistically significant $(P<0.05)$; The CFS score of the two groups was lower than that before intervention, and that of the observation group was lower than that of the control group, the difference was statistically significant $(P<0.05)$. Conclusion: KAP intervention model can improve the resilience of patients with colorectal cancer chemotherapy, reduce cancerrelated fatigue.
\end{abstract}

Key words: Colorectal cancer; Knowledge, attitude and practice model; Psychological flexibility; Cancer related fatigue

Publication date: January, 2021

Publication online: 31 January, 2021

"Corresponding author: Hui Zhang,

\section{Introduction}

Surgical resection and chemotherapy are often used in the treatment of colorectal cancer in order to improve the clinical symptoms such as bloody stool and change of defecation habits, and prolong the survival period of patients. However, due to the fear of cancer and the side effects of chemotherapy drugs, patients often feel physically and mentally tired and lose confidence in treatment, so effective measures should be taken as soon as possible. Knowledge attitude practice (KAP) intervention mode mainly through knowledge, belief and behavior and other aspects of intervention, gradually change patients' uncertain behavior, so as to develop healthy behavior ${ }^{[1]}$. In order to promote the rehabilitation of patients, this study mainly discusses the effect of KAP intervention mode on psychological resilience and cancer-related fatigue of patients with colorectal cancer chemotherapy. It is shown as follows.

\section{Material and methods}

\subsection{General information}

This study was approved by the medical ethics committee of our hospital [2018 s (095)]. 55 patients with colorectal cancer who received routine nursing from February 2018 to February 2019 were included in the control group, and 55 patients who received routine nursing + KAP intervention from March 2019 to March 2020 were included in the observation group. There were 32 males and 23 females in the observation group; The average age was (45.32 \pm 3.77) years old; The course of disease was 1-2 years, 
with an average of $(1.53 \pm 0.17)$ years; Location of the lesion: There were 33 cases of colon and 22 cases of rectum. There were 34 males and 21 females in the control group; The average age was $(45.81 \pm 3.83)$ years old; The course of disease was $1-2$ years, with an average of $(1.55 \pm 0.18)$ years; Location of the lesion: There were 35 cases of colon and 20 cases of rectum. The general data of the two groups were comparable $(P>0.05)$.Patients and their families voluntarily signed informed consent.

\subsection{Inclusion criteria}

(1) inclusion criteria: According to the diagnostic criteria of colorectal cancer in Chinese code for diagnosis and treatment of colorectal cancer ${ }^{[2]}$; The cognitive ability is normal, with reading and writing ability; The estimated survival time was more than 6 months. Exclusion criteria: combined with other malignant tumors; Complicated with cardiopulmonary dysfunction; The patients were treated with related drugs one month before chemotherapy.

\subsection{Method}

\subsubsection{The control group}

Routine nursing intervention: nursing staff simply popularize knowledge of colorectal cancer disease for patients; Give medication and later treatment guidance; Telephone follow-up once a month to understand the patient's condition, ask the patient to review regularly, and continue to intervene for 6 months.

\subsubsection{The observation group}

KAP intervention mode was adopted on the basis of the control group: a KAP intervention group was established, consisting of the head of the department, the head nurse and 3 responsible nurses. After meeting and discussion, the group members developed targeted measures according to the patient's condition. The head nurse and department director are responsible for giving professional guidance and training to the responsible nurses, and providing targeted nursing for patients after passing the assessment. The details are as follows:(1) Strengthen knowledge education. Arrange lectures on colorectal cancer knowledge, invite oncology experts to explain the etiology, treatment and prognosis of colorectal cancer, and answer questions from patients and their families; The nursing Manual of colorectal cancer was issued, and related health education articles and videos were pushed to patients through wechat platform every day to improve their disease awareness. (2) Build faith: Encourage patients to explain their psychological demands, and suggest their families to accompany patients more; Explain the doubts of patients, list successful treatment cases, and increase the patients' belief in conquering the disease; Guide patients to use appropriate methods, such as deep breathing, listening to music, muscle relaxation, etc., to transfer patients' anxiety.(3) Reinforcement behavior: To guide patients to carry out reasonable aerobic exercise, including walking, broadcast gymnastics, Taijiquan, etc., with the patient's maximum tolerance, 90 minutes each time, 5 times a week; And weekly through wechat to guide patients' medication, diet, colostomy care, etc., encourage patients to adhere to self-care, and instruct patients' families to supervise their implementation. The intervention lasted for 6 months.

\subsection{Evaluation index}

Mental resilience: Mental resilience scale ${ }^{[3]}$ was used to evaluate the psychological changes of the two groups, including three dimensions of resilience, selfimprovement and optimism. There were 25 items in total, and each item was scored according to the 1-4 grading method with a total of 100 points. The higher the score, the better the mental resilience. Cancer related fatigue: Cancer fatigue scale (CFS) ${ }^{[4]}$ was used to evaluate the three dimensions of physical fatigue, emotional fatigue and cognitive fatigue, with 15 items in total. Each item scored 0-4, and the total score was $0-60$. The higher the score, the more serious the cancer-related fatigue.

\subsection{Statistical methods}

SPSS 24.0 software was used to process data. to represent measurement data. Independent sample t-test was used between groups, paired sample t-test was used within groups, and count data was expressed as\%. $\chi^{2}$ test was used. $P<0.05$ was considered as statistically significant.

\section{Results}

Before intervention, there was no significant difference in resilience and CFS score between the two groups $(P>0.05)$; After 6 months of intervention, the elasticity score of the two groups was higher than 
that before intervention, and the observation group was higher than that of the control group, the CFS score of the two groups was lower than that before intervention, and the observation group was lower than that of the control group, the difference was statistically significant $(P<0.05)$. See Table 1 .

Table 2. Comparison of resilience and CFS scores between the two groups $(\bar{x} \pm s$, points $)$

\begin{tabular}{|c|c|c|c|}
\hline Time & Group & Resilience score & CFS score \\
\hline \multirow{4}{*}{ Before intervention } & Observation group $(n=55)$ & $56.31 \pm 4.56$ & $38.74 \pm 3.67$ \\
\hline & Control group $(n=55)$ & $56.33 \pm 4.57$ & $39.16 \pm 3.71$ \\
\hline & $t$ & 0.023 & 0.597 \\
\hline & $P$ & 0.982 & 0.552 \\
\hline \multirow{3}{*}{ The intervention lasted for 6 months } & Observation group $(n=55)$ & $73.99 \pm 5.63^{\mathrm{a}}$ & $25.68 \pm 2.75^{\mathrm{a}}$ \\
\hline & Control group $(n=55)$ & $63.10 \pm 5.31^{\mathrm{a}}$ & $22.38 \pm 1.56^{\mathrm{a}}$ \\
\hline & $P$ & $<0.001$ & $<0.001$ \\
\hline
\end{tabular}

Note: Compared with the same group before intervention, ${ }^{\mathrm{a}} P<0.05$

\section{Discussion}

Patients with colorectal cancer are prone to fear, tension and other negative emotions under the influence of disease torture and side effects of chemotherapy drugs, and lose confidence in treatment, which affects the recovery of the disease. Therefore, effective nursing intervention for patients with colorectal cancer chemotherapy is particularly important.

KAP intervention mode improves patients' belief in treatment by increasing patients' cognition of disease, and actively adopts healthy behaviors under the guidance of belief, so as to achieve the purpose of promoting patients' rehabilitation ${ }^{[5]}$. The results showed that after 6 months of intervention, the resilience score of the observation group was higher than that of the control group, suggesting that KAP intervention model can improve the resilience of patients with colorectal cancer chemotherapy. The reason is that the KAP intervention group can improve the patients' awareness of the disease by arranging expert lectures, distributing brochures and wechat push.

The results of this study showed that the CFS score of the observation group was lower than that of the control group after 6 months of intervention, suggesting that KAP intervention mode can improve cancer-related fatigue in patients with colorectal cancer chemotherapy. The reason is that cancerrelated fatigue can lead to physical and mental fatigue, make patients give up treatment, which is not conducive to the recovery of patients. KAP intervention mode can deepen patients' cognition of colorectal cancer, improve patients' confidence in treatment, and help patients establish awareness of healthy behavior through various forms of targeted education; Guiding patients to exercise reasonably, and guiding medication and nursing through wechat, family supervision and encouragement can strengthen patients' health behavior and reduce cancer-related fatigue.

In conclusion, KAP intervention model can improve the psychological resilience of patients with colorectal cancer chemotherapy and reduce cancerrelated fatigue.

\section{References}

[1] He XX, Yang HJ, Yan YY. Application of zhixinxing in improving the compliance of review of postoperative patients with colorectal cancer[J]. Guangdong Medical Journal, 2016, 37(19): 2993-2995

[2] Oncology branch of Chinese Medical Association, national health and Family Planning Commission, medical administration and hospital administration. Chinese standard for diagnosis and treatment of colorectal cancer (2017 Edition) [J]. Chinese Journal of gastrointestinal surgery, 2018, 21 (1): 92-106

[3] Ni QY, Tian J. Reliability and validity evaluation and application of Resilience Scale [J]. China public health, 2013, 29 (10): 1524-1527

[4] Zhang FL, Ding Y, Han LS. Reliability and validity of Chinese version of cancer fatigue scale $[\mathrm{J}]$. Chinese Journal of mental health, 2011, 25 (11): 16-19

[5] Dai MZ, Xia LM, Zuo ZG, et al. Effect of palliative care management based on KAP theory on patients with advanced colorectal cancer undergoing chemotherapy [J]. China Medical Journal, 2020, 17(1): 185-188. 